BULL. AUSTRAL. MATH. SOC.

$60 J 70,92 A 10$

VOL. 9 (1973), 311.

\title{
Topics in population genetics
}

\section{Beverley June Balkau}

This thesis deals with a number of independent topics in theoretical population genetics. A summary of the topics treated is given below, chapter by chapter.

The first four chapters are concerned with the modification of linkage, by a selectively neutral locus, in deterministic genetic models. The general result, obtained for randomly mating and selfing diploids, and for haploids mating under an incompatibility mechanism, is that, given a stable two locus polymorphism, there can be 'secondary selection' to reduce the recombination frequency between these loci. Under certain conditions in the selfing and randomly mating populations, a polymorphism can exist at the recombination modifying locus, if the heterozygote at the modifying locus causes tighter linkage than either of the homozygotes.

Chapter 5 shows that the migration rate between population can be reduced, if migration rate is controlled by a selectively neutral locus. A two locus sex-linked model is formulated in Chapter 6 , and equilibria and stability conditions determined for a particular symmetric set of fitnesses. In Chapter 7, the substitutional load for two linked loci is calculated numerically for a deterministic haploid model, and the effects of linkage and epistasis noted. In Chapter 8, diffusion methods are used to determine the absorption probability of an allele in a haploid population in which the allele has a selective advantage or disadvantage with probabilities $\pi$ and $1-\pi$. The final chapter applies some methods of branching processes to multiallelic populations, and to populations of changing size.

Received 27 June 1973. Thesis submitted to La Trobe University, February 1973. Degree approved, June 1973. Supervisors: Dr M.W. Feldman, Professor W.J. Ewens. 